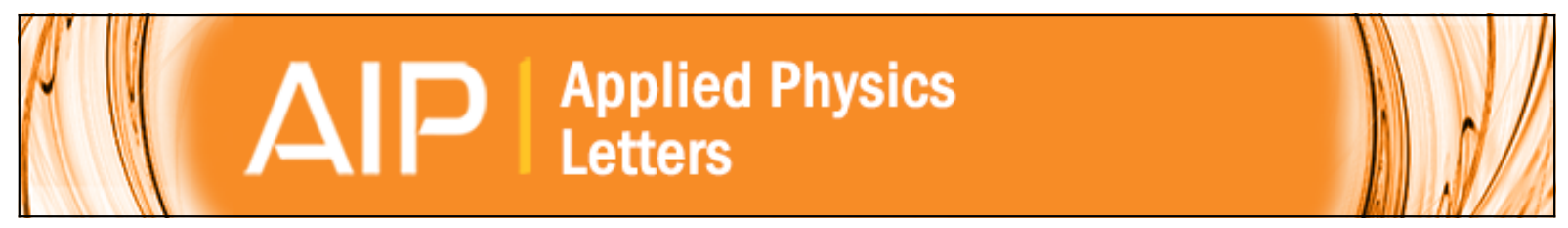

\title{
Extraordinary optical transmission with coaxial apertures
}

Shannon M. Orbons, Ann Roberts, David N. Jamieson, Michael I. Haftel, Carl Schlockermann, Darren Freeman, and Barry Luther-Davies

Citation: Applied Physics Letters 90, 251107 (2007); doi: 10.1063/1.2751120

View online: http://dx.doi.org/10.1063/1.2751120

View Table of Contents: http://scitation.aip.org/content/aip/journal/apl/90/25?ver=pdfcov

Published by the AIP Publishing

\section{Articles you may be interested in}

Extraordinary transmission through a silver film perforated with bowtie-shaped aperture array in midinfrared region

Appl. Phys. Lett. 98, 253107 (2011); 10.1063/1.3599851

Phaselike resonance behavior in optical transmission of sandwich coaxial square ring arrays

Appl. Phys. Lett. 96, 253102 (2010); 10.1063/1.3442498

Excitation of dielectric-loaded surface plasmon polariton observed by using near-field optical microscopy Appl. Phys. Lett. 93, 073306 (2008); 10.1063/1.2973355

Transmission of light by a single subwavelength cylindrical hole in metallic films

Appl. Phys. Lett. 89, 141110 (2006); 10.1063/1.2358210

Refractive transmission of light and beam shapingwith metallic nano-optic lenses

Appl. Phys. Lett. 85, 642 (2004); 10.1063/1.1776327

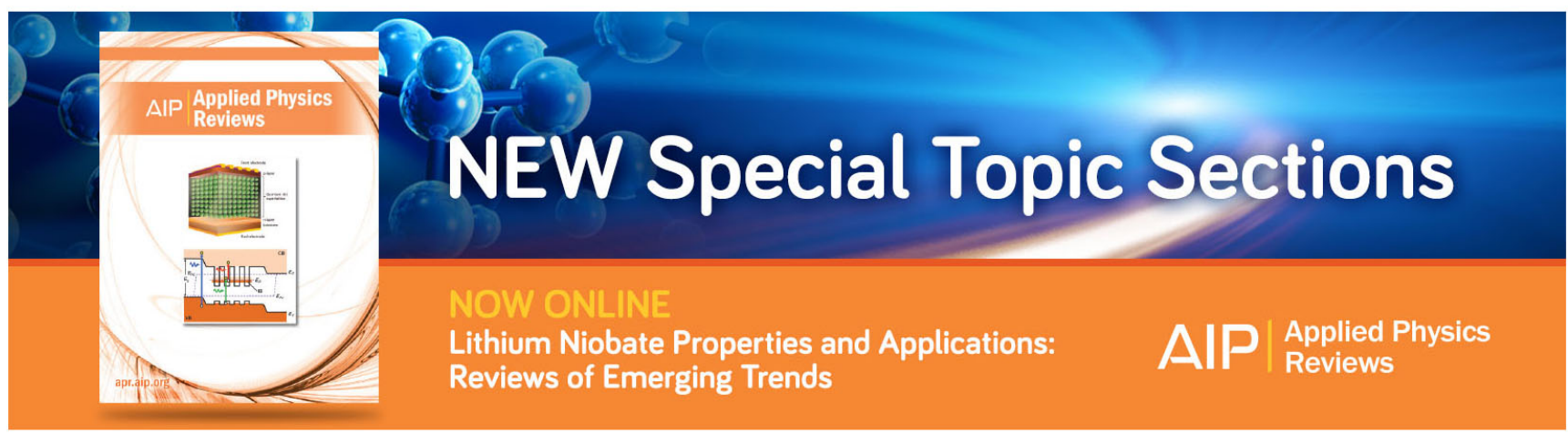




\title{
Extraordinary optical transmission with coaxial apertures
}

\author{
Shannon M. Orbons, ${ }^{\text {a) }}$ Ann Roberts, and David N. Jamieson \\ School of Physics, University of Melbourne, Melbourne, Victoria 3010, Australia \\ Michael I. Haftel \\ Center for Computational Materials Science, Naval Research Laboratory, Washington, DC 20735-5343
}

Carl Schlockermann

I. Physikalisches Institut (IA), RWTH Aachen, 52056 Aachen, Germany

Darren Freeman and Barry Luther-Davies

Centre for Ultrahigh-Bandwidth Devices for Optical Systems, Laser Physics Centre, Australian National University, Canberra, Australian Capital Territory 0200, Australia

(Received 26 March 2007; accepted 31 May 2007; published online 20 June 2007)

\begin{abstract}
Recently it has been predicted that "cylindrical" surface plasmons (CSP's) on cylindrical interfaces of coaxial ring apertures produce a different form of extraordinary optical transmission that extends to ever increasing wavelengths as the dielectric ring narrows. This letter presents experimental confirmation of this CSP assisted extraordinary transmission. Nanoarrays of submicron coaxial apertures are fabricated in a thin silver film on a glass substrate and far-field transmission spectra are measured. The experimental spectrum is in close agreement with predictions from finite-difference time-domain simulations and CSP dispersion theory. The role of cylindrical surface plasmons in producing extraordinary transmission is thus confirmed. (c) 2007 American Institute of Physics.
\end{abstract}

[DOI: $10.1063 / 1.2751120]$

The role of surface plasmons (SP's) in extraordinary optical transmission ${ }^{1}$ (EOT) is controversial. The original work $^{1}$ of Ebbesen et al. attributes the enhanced optical transmission through nanoarrays of subwavelength apertures in metal films to SP's (Refs. 1-3) on the surfaces of the metal film. However, other investigators ${ }^{4-8}$ have shown that certain shape and diffractive effects produce EOT on perfectly conducting or semiconducting films without surface plasmons. The explicit role of SP's has not been completely resolved since the other enhancements often occur at frequencies very close to those where surface plasmons would be launched.

Several investigators ${ }^{9-12}$ have recently pointed out a unique role for nanoarrays of coaxial apertures in metallic films in EOT. Finite-difference time-domain (FDTD) simulations $^{9-11}$ indicate that the transmission peaks from such arrays are considerably redshifted from those of cylindrical apertures of the same diameter. Theoretical analyses by Baida et al. ${ }^{10}$ and Haftel et al. ${ }^{11}$ show that this redshifting is due to SP's propagating on the cylindrical metal-dielectric (or metal-vacuum) interfaces of these apertures, i.e., "cylindrical" surface plasmons (CSP's). Furthermore, the longwavelength enhancements are due to propagating modes through the individual coaxial rings (CR's) with the nanoarray periodicity playing only a minor role. Somewhat counterintuitively, the cutoff wavelength for propagating modes increases indefinitely as the ring narrows, ${ }^{11}$ which is a direct consequence of the dielectric constant of the metal being negative with only a small imaginary part. This CSP enhancement mechanism is distinct from earlier forms of EOT (Refs. 1-3) attributable to "planar" surface plasmons (PSP's) propagating on the surfaces of the metal films, which channel the electromagnetic energy more efficiently into the apertures enhancing what are still evanescent waves.
In this letter we confirm CSP enhanced extraordinary transmission from CR apertures by comparing experimental measurements with FDTD simulations and theoretical predictions. ${ }^{10,11}$ To date, Salvi et al. ${ }^{13}$ have fabricated nanoarrays of such apertures and conducted measurements of the transmission spectrum. While measurements and FDTD simulations in this work agree fairly well, the transmission spectrum was measured up to only $900 \mathrm{~nm}$, whereas an unambiguous CSP transmission peak is predicted to be at $1330 \mathrm{~nm}$. Orbons et al. ${ }^{14}$ have measured up to $1700 \mathrm{~nm}$ for similar apertures, which exceeds the wavelengths where CSP effects should appear, but their published simulations assume perfect conductivity within the metal and do not quantitatively predict the CSP peak position.

Utilizing CSP dispersion relations and FDTD simulations, Refs. 10 and 11 predict, for metal films with Drudelike dispersion, the following. (1) A CSP transmission peak occurs whenever a cylindrical CSP $n=1$ normal mode is supported for an isolated aperture when the longitudinal wave number $k_{z}$ satisfies $k_{z} L=m \pi$, where $m$ is an integer and $L$ is the length of the aperture. Thus these are propagating (predominantly $\mathrm{TE}_{1}$ ) modes, not evanescent, through the aperture. (2) The peak at the longest wavelength is for $m=0$ and corresponds to $k_{z}=0$, i.e., at the cutoff wavelength, and its position is independent of $L$. (3) The peak position is approximately independent of the array periodicity. (4) In the visible and IR the wavelength of the CSP peaks increases indefinitely as $\left(R_{2}-R_{1}\right)^{-1 / 2}$, where $R_{1}$ and $R_{2}$ are the inner and outer radii of the $\mathrm{CR}$, respectively.

Figure 1 illustrates some of the predicted trends of CSP assisted extraordinary transmission. Here we simulate the coaxial apertures of the type fabricated by Orbons et al. ${ }^{14}$ using FDTD. The apertures consist of a silver core embedded in a silver film of thickness $L=140 \mathrm{~nm}$ deposited on a glass substrate and have outer radii of $290 \mathrm{~nm}$ with periodicity $(900 \mathrm{~nm})^{2}$. Losses are negligible for the wavelengths and

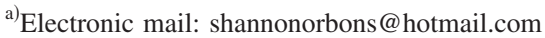




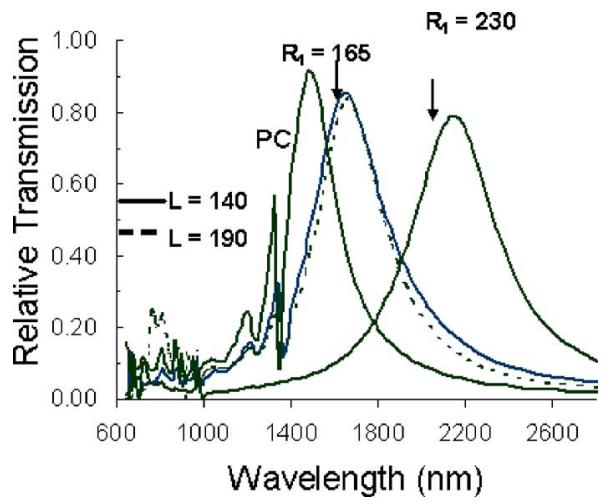

FIG. 1. (Color online) FDTD transmission spectra for the CR arrays of Ref. 14. Results for a perfect conductor (PC) are included. The theoretical positions of cylindrical surface plasmon (CSP) resonances are indicated, as well as the inner radii and CR lengths (in nanometers).

geometry of interest, with the CSP range $>50 \mu \mathrm{m}$. When the inner radius is increased from 165 to $230 \mathrm{~nm}$, the transmission peak is increased from 1638 to $2091 \mathrm{~nm}$, close to the $\left(R_{2}-R_{1}\right)^{-1 / 2}$ rule. The theoretical peak positions, calculated from CSP dispersion curves ${ }^{11}$ are 1656 and $2166 \mathrm{~nm}$, respectively, as shown. Figure 1 includes results for $R_{1}=165 \mathrm{~nm}$ and $L=140 \mathrm{~nm}$ when silver is replaced by a perfect conductor (PC). Here peaks appear at 1489 and $1034 \mathrm{~nm}$. Theoretically, the PC cutoff should be $\lambda_{\text {cutoff }} \approx \pi\left(R_{1}+R_{2}\right)=1555 \mathrm{~nm}$, ${ }^{15}$ which corresponds to an $m=0$ peak, and the planar surface plasmon peak should be at $993 \mathrm{~nm}$. Thus the simulated peaks for the PC case are near the theoretical positions, and the redshifting of the $m=0$ peak for $\mathrm{Ag}$ relative to a $\mathrm{PC}$ exhibits the role of the dielectric function of the metal.

Details of the fabrication of the CR aperture arrays and optical measurements have been outlined elsewhere. ${ }^{14}$ Figure 2 shows a scanning electron microscopy image of an array of annular apertures in a $140 \mathrm{~nm}$ thick film, with a period of $900 \mathrm{~nm}$ and inner and outer diameters of 330 and $580 \mathrm{~nm}$, respectively. To measure the normal incidence transmission spectrum of this device, the sample was illuminated using a supercontinuum source outlined in Ref. 14. Due to the coherent nature of the incident radiation, the Fabry-Pérot fringes are present in the raw experimental data caused by interference within the glass substrate. The experimental data shown in Fig. 3 have been filtered to remove these fringes.

The main results of our investigation appear in Fig. 3, which compares the experimental measurements for the $R_{1}=165 \mathrm{~nm}$ and $L=140 \mathrm{~nm}$ apertures with the FDTD simu-

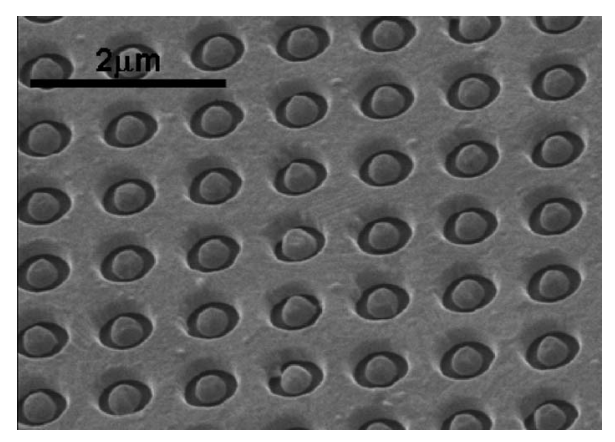

FIG. 2. Images of a $900 \mathrm{~nm}$ period $100 \times 100$ array of annular apertures with inner and outer diameters of 330 and $580 \mathrm{~nm}$, respectively, in a $140 \mathrm{~nm}$ thick silver film on a glass substrate.

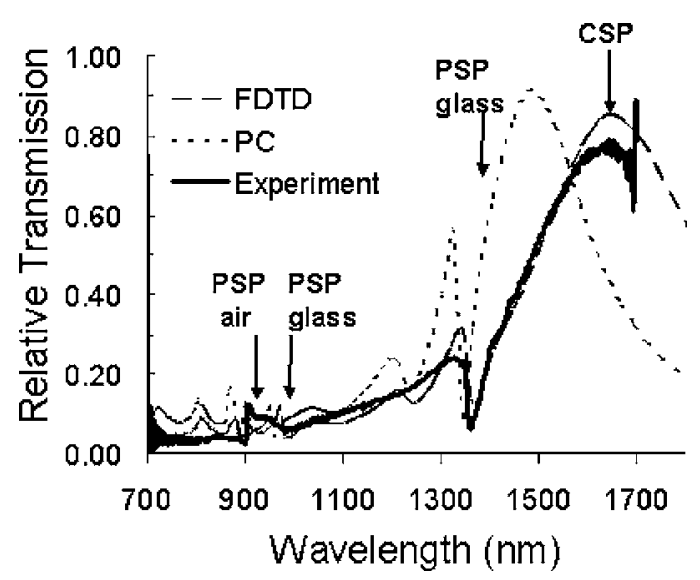

FIG. 3. Experimental and FDTD transmission spectra for the arrays of CR apertures of Ref. $14\left(R_{1}=165 \mathrm{~nm}, R_{2}=290 \mathrm{~nm}\right.$, and $\left.L=140 \mathrm{~nm}\right)$. Results for a PC are included. Theoretical positions of CSP and PSP resonances are shown.

lations. The results for Ag replaced by a PC are repeated for reference. This figure also indicates the theoretical position of the CSP peak for such an aperture as well as the positions of the PSP resonances, for $(900 \mathrm{~nm})^{2}$ periodicity, at the glass-metal interface and air-metal interface at the surfaces of the film.

The simulation and experiment are in very good agreement, especially with respect to the peak position and its shape. The measured peak at $1659 \mathrm{~nm}$ is close to the simulated peak at $1638 \mathrm{~nm}$ and very close to the theoretical CSP resonance at $1656 \mathrm{~nm}$. This differs substantially from the PC peak at $1489 \mathrm{~nm}$ and provides strong confirmation of the predictions ${ }^{10,11}$ on the role of CSP's. The peak-dip structure at $1300-1350 \mathrm{~nm}$ appears very similar in experiment and simulation, these being associated with Wood's anomaly and a PSP $(1,0)$ resonance, which theoretically should be at 1367 and $1385 \mathrm{~nm}$, respectively, but are slightly blueshifted from these positions in both experiment and simulation. The agreement between experiment and simulation generally persists over the full spectral range, with the simulation exhibiting somewhat more structure than indicated in experiment. Except for the CSP peak, the simulated Ag and PC results give similar structure with most of the features being attributable to PSP and diffractive effects.

In summary, this investigation provides experimental demonstration of CSP assisted extraordinary optical transmission and the role of coaxial apertures in its enhancement. There are several important consequences. Whereas the cutoff for PC CR apertures is limited to a constant value $2 \pi R_{2}$ as $R_{1} \rightarrow R_{2}$, the cutoff for a real metal $\mathrm{CR} \sim\left(R_{2}-R_{1}\right)^{-1 / 2}$. Since the CSP enhancement mechanism relies on propagating modes, the transmitted intensity of $\mathrm{CR}$ apertures with narrow rings should fall off no faster than the exposed area of such rings. Indeed, the previous study ${ }^{11}$ indicated that the transmitted intensity was two to four times the intensity falling on the exposed rings, whereas the present study indicates a similar enhancement factor of 3-4. Even off resonance $\left(k_{z} L \neq m \pi\right)$ the increased cutoff from CR's will promote what would otherwise be evanescent waves in the aperture to propagating waves (or at least to less rapidly decaying evanescent waves), and this will enhance transmission.

At lower frequencies where surface plasmons extend very far from the interfaces and become very diffuse (far 
infrared and lower), the CSP enhancement effect will die out. However, recent research ${ }^{16-18}$ has shown that subwavelength periodic dielectric structures embedded in perfect conductors can produce "spoof" surface plasmons, i.e., electromagnetic waves bound to the interfaces of such materials with homogeneous dielectrics. We are now exploring the possibility of designing such CSP metamaterials for rf and microwave frequencies.

This work was partially supported by the Office of Naval Research. Computations were carried out under the Department of Defense High Performance Computation Modernization Project. The support of the Australian Research Council through its Centers of Excellence, Federation Fellow and Discovery programs is gratefully acknowledged. The authors also thank the Australian National University's Electron Microscopy Unit for the use of the FIB.

${ }^{1}$ T. W. Ebbesen, H. J. Lezec, H. F. Ghaemi, T. Thio, and P. A. Wolff, Nature (London) 391, 667 (1998).

${ }^{2}$ L. Martín-Moreno, F. J. García-Vidal, K. M. Pellerin, T. Thio, J. B. Pendry, and T. W. Ebbesen, Phys. Rev. Lett. 86, 1114 (2001).

${ }^{3}$ W. L. Barnes, W. A. Murray, J. Dintinger, E. Devaux, and T. W. Ebbesen, Phys. Rev. Lett. 92, 107401 (2004).
${ }^{4}$ K. J. Klein Koerkamp, S. Enoch, F. B. Segerink, N. F. van Hulst, and L. Kuipers, Phys. Rev. Lett. 92, 183901 (2004).

${ }^{5}$ R. Gordon and A. G. Brolo, Phys. Rev. Lett. 92, 037401 (2004).

${ }^{6}$ J. Bravo-Abad, F. J. García-Vidal, and L. Martín-Moreno, Phys. Rev. Lett. 93, 227401 (2004).

${ }^{7}$ G. P. Wang, Y. Yi, and B. Wang, J. Phys.: Condens. Matter 15, 8147 (2003).

${ }^{8}$ H. J. Lezec and T. Thio, Opt. Express 12, 3629 (2004).

${ }^{9}$ F. I. Baida and D. van Labeke, Phys. Rev. B 67, 155314 (2003); F. I. Baida, D. Van Labeke, G. Granet, A. Moreau, and A. Belkhir, Appl. Phys. B: Lasers Opt. 79, 1 (2004).

${ }^{10}$ F. I. Baida, A. Belkhir, D. Van Labeke, and O. Lamrous, Phys. Rev. B 74, 205419 (2006).

${ }^{11}$ M. I. Haftel, C. Schlockermann, and G. Blumberg, Appl. Phys. Lett. 88, 193104 (2006); Phys. Rev. B 74, 235405 (2006).

${ }^{12}$ S. M. Orbons and A. Roberts, Opt. Express 14, 12623 (2006).

${ }^{13}$ J. Salvi, M. Roussey, F. I. Baida, M.-P. Bernal, A. Mussot, T. Sylvestre, H. Maillotte, D. Van Labeke, A. Perentes, I. Utke, C. Sandu, P. Hoffman, and B. Dwir, Opt. Lett. 30, 1611 (2005).

${ }^{14}$ S. M. Orbons, D. Freeman, B. C. Gibson, S. T. Huntington, B. Luther-Davies, D. N. Jamieson, and A. Roberts, Physica B 394, 176 (2007).

${ }^{15}$ A. W. Snyder and J. D. Love, Optical Waveguide Theory (Chapman and Hall, London, 1983).

${ }^{16}$ J. B. Pendry, L. Martin-Moreno, and F. J. Garcia-Vidal, Science 305, 847 (2004).

${ }^{17}$ F. J. García de Abajo and J. J. Sáenz, Phys. Rev. Lett. 95, 233901 (2005).

${ }^{18}$ A. P. Hibbins, B. R. Evans, and J. R. Sambles, Science 308, 670 (2005). 